\title{
Epigenetic cues emerging from lysine acetylation bridge chromatin-mediated transcription to mitochondrial functions
}

\begin{abstract}
The landscape of lysine acetylation has expanded from nucleus to a cellular-wide global event. The substrates of histone acetyltransferase (HAT) in addition to chromatin and transcription factors now include metabolic enzymes, cytoskeletal proteins, molecular chaperones, ribosomal proteins and nuclear import factors. Together, lysine acetylation is involved in regulating protein stability, chromatin-mediated transcriptional activation, subcellular localization and activities of metabolic enzymes. Growing number of investigations reveal that a significant number $(2000)$ of proteins are undergoing acetylation on either one or more than one lysine residues. Notably, our review underscores the dynamic nature of acetylated lysine site (s), which can become a recruitment site(s) for bromodomain-containing proteins or histone deacetyltransferases that ultimately facilitates cellular response to diverse internal and external environmental changes. Finally, our review emphasizes on the strength of small chemical molecules, which do not perturb the expression of endogenous proteins to address complexities of epigenetic modifications. These small molecules can serve as tools to enhance our mechanistic insights as well as have therapeutic potential to treat many diseases.
\end{abstract}

Keywords: epigenetics, lysine acetylation, deacetylation, chromatin, transcription, metabolism
Volume 2 Issue 2 - 2015

Zimuzoh Orakwue, Shiraz Mujtaba

Department of Biology, City University of New York, USA

Correspondence: Shiraz Mujtaba, Department of Biology, Medgar Evers College, City University of New York, 1638 Bedford Ave, Brooklyn, NY I I225, USA, Tel 718270 6191, Fax 718270 6196, Email smujtaba@mec.cuny.edu

Received: May 01, 2015 | Published: May 19, 2015
Abbreviations: HAT, histone acetyl transferase; HDACs, histone deacetylase; FDA, food and drug administration

\section{Introduction}

Amongst 20 amino acids, lysine is the most versatile owing to its steric flexibility, which facilitate several chemical modifications, such as acetylation, butyrylation, crotonlylation, formylation, hydroxylation, malonylation, methylation, propionylation, ubiquitination, succinylation and sumoylation. ${ }^{1-14}$ Though serine/ threonine phosphorylation and lysine acetylation were discovered between 1959 and 1963 respectively, most investigations focused on understanding the functions of kinases during that time. ${ }^{15,16}$ Two major studies that propelled unraveling the significance of acetylation include the discovery of coenzyme A and acetyl-CoA, followed by the evidence that acetylation of histone proteins could modulate gene transcription. ${ }^{17,18}$ Growing number of studies have established that lysine acetylation plays a crucial role in the pathogenesis of diseases, including diabetes, obesity, inflammation and cancers. ${ }^{19-22}$ Essentially, acetylation not only imparts gene regulatory capabilities to the transcriptional machinery, but it also governs cellular processes not limited to DNA replication, DNA damage and repair, chromatin remodeling, metabolism and cell cycle progression. ${ }^{23-27}$

Hereafter, the notion that acetylation is restricted to modulating chromatin-mediated transcription function expanded and now, it is recognized to be a global event involving mitochondrial and cytosolic proteins. ${ }^{28}$ Consequently, lysine acetylation is not only limited to delivering plasticity to the chemical landscape of chromatin, but it also affects stability, sub-cellular localization and molecular interactions of proteins..$^{29}$ Additionally, the physiological and cellular roles of auto acetylation remain to be fully understood. ${ }^{30}$ Transition from investigating the role of acetylation during chromatin-mediated transcriptional regulation to deciphering the significance of acetylation on the functions of transcription factors, such as p53, established the epigenetic basis of gene regulation during cellular response to stress. The major impact of these findings navigated the elucidation of acetylation -mediated molecular interactions with bromodomain and dynamic regulation of acetylation/deacetylation by histone deacetylase (HDACs) (Figure 1). ${ }^{31}$ Furthermore, the identification of NAD -depended sirtuins in mitochondria indicated that acetylation could regulate metabolic events also. ${ }^{31}$ In 2009, Mann and his colleagues using high-resolution mass spectrometry discovered that there are atleast 3,600 acetylated lysine sites (Kac) sites in 1,750 cellular proteins suggesting acetylation of multiple lysine residues on a single protein. ${ }^{1,28}$ Notably, about 500 acetylated proteins participate in the chromatin-templated processes. Out of which, approximately 50 acetylated proteins are most likely involved in the process of DNA replication and another 80 could potentially play a crucial role during the process of DNA damage and repair. ${ }^{1,28}$ Similarly, about 40 acetylated proteins could be involved in regulating of cell cycle genes and approximately, 60 proteins in nucleotide exchange factors. ${ }^{1,28}$

Depending upon cellular environment conditions, about 55 proteins, which are involved in DNA/RNA helicase activity and nearly, 56 proteins possessing ubiquitin ligase and deubiqutinases activities could undergo acetylation. ${ }^{1,28}$ Given that multiple lysine residues are acetylated on a protein raises the possibility of a combinatorial pattern of acetylation, which could serve as a signature for facilitating cellular response to a diverse varieties of stress. ${ }^{28}$ During gene transcription, 
one of the major challenges is to define acetylation-specific outcomes mainly because most histone acetyltransferases (HATs) are comprised of multi-domain proteins, which are capable of executing more than one function at a given time..$^{32}$ For instance, p300 and CBP are comprised of multiple cysteine-rich moieties, plant like homeodomain and bromodomain in addition to HAT. Similarly, MYST1 is composed of chromodomain that binds to methylated-lysine as well as a HAT domain which acetylate human histone $\mathrm{H} 4$ on lysine $16 .{ }^{33}$ TAFII250 coactivator contains double bromodomain, a HAT as well as Kinase domains. ${ }^{34}$ Clearly, due to these multitude of activities associated with a particular HAT, overexpression as well as genetic knockout strategies may not fully convey the acetylation-specific outcomes. Furthermore, depending upon the alterations of external and internal environmental conditions, lysine acetylation could be mutually exclusive to methylation or ubiquitination. Collectively, these data underline that epigenetic modifications could be best studied by using small molecules, which does not perturb the expression of endogenous proteins. In this direction, the development of acetylation-specific antibodies has been one of the most effective tools that enhanced our understanding of the kinetics driving acetylation. ${ }^{35}$ With the advent of proteomic and genomic high throughput technologies knowledge of new molecular targets that modulate gene functions have tremendously increased. Particularly, the dynamism exhibited by epigenetic modifications, such as acetylation/deacetylation, which if perturbed by a small molecule has potential to impact cell fate decisions that eventually has made them attractive targets for drug discovery program.

\section{Epigenetic Regulation}

\section{Metabolic Regulation}

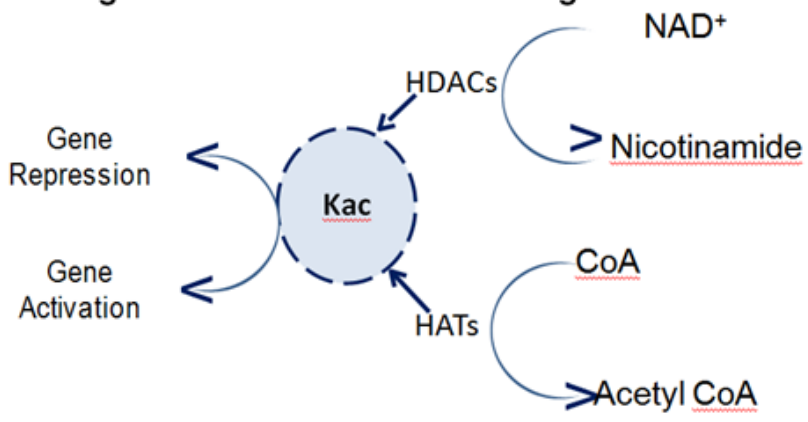

Figure I The amino acid lysine can be acetylated (Kac) by histone acetyltransferases (HATs) and deacetylated by HDACs. Both HATS and HDAcs are dependent upon cofactors, which are pivotal to metabolic pathway. Since dynamics of acetylation/deacetylation also regulates chromatin -mediated gene transcription, an acetylated lysine is a focal point of regulation for key cellular processes.

The FDA-approved HDAC inhibitor, Vorinostat, induces the differentiation of tumor cells has been approved for treatment of $\mathrm{T}$ cell lymphoma. ${ }^{36}$ Besides, several inhibitors of bromodomain showed promising results in blocking pathogenesis of ischemia and HIV infection..$^{37,38}$ Most recently, inhibitor of BRD4, JQ1, which was tested in various model systems, suppresses $\mathrm{CMYC}$ by binding to the first bromodomain of BRD $4 .{ }^{39}$ Clearly, emerging studies show promising small molecules that target HDACs or bomodomain, but a selective ligand for histone acetyltransferase remains to be developed. Most importantly, chemical biology is a gateway for not only dissecting the function of an endogenous protein with multiple functions but also could offer highly selective ligand which could have therapeutic value. Taken together, the ability of amino acid lysine to facilitate quick chemical alterations that aid cellular capability to adjust as well as respond to environmental changes upon genomic stress, viral infection and energy generating metabolic reactions is remarkable. Given the genome-wide numerical ratio of HATs to HDACs or to Sirts as well as to bromodomains, it appears that we have only begun to scratch at the surface to understand the complete biological significance of acetylation in maintaining the cellular homoeostasis. However, we take this opportunity to salute investigators contributing through 50years since the discovery of acetylation.

\section{Acknowledgements}

We deeply appreciate help from the members of writing center at Medgar Evers College for critically reading our article. We are deeply grateful to NIGMS-RISE program at MEC for supporting ZO and to NCI for supporting SM.

\section{Conflict of interest}

Author declares that there is no conflict of interest.

\section{References}

1. Choudhary C, Kumar C, Gnad F, et al. Lysine acetylation targets protein complexes and co-regulates major cellular functions. Science. 2009;325(5942):834-840.

2. Van Slyke DD, Sinex FM. The course of hydroxylation of lysine to form hydroxylysine in collagen. J Biol Chem. 1985;232(2):797-806.

3. Chen Y, Sprung R, Tang Y, et al. Lysine propionylation and butyrylation are novel post-translational modifications in histones. Mol Cell Proteomics. 2007;6(5):812-819.

4. Tan M, Luo H, Lee S, et al. Identification of 67 histone marks and histone lysine crotonylation as a new type of histone modification. Cell. 2011;146(6):1016-1028.

5. Hochstrasser M. Origin and function of ubiquitin-like proteins. Nature. 2009;458(7237):422-429.

6. Kim W, Bennett EJ, Huttlin EL, et al. Systematic and quantitative assessment of the ubiquitin-modified proteome. Mol Cell. 2011;44(2):325-340.

7. Wagner SA, Beli P, Weinert BT, et al. A proteome-wide, quantitative survey of in vivo ubiquitylation sites reveals widespread regulatory roles. Mol Cell Proteomics. 2011;10(10):M111.

8. Wisniewski JR, Zougman A, Mann M. Nepsilon-formylation of lysine is a widespread post-translational modification of nuclear proteins occurring at residues involved in regulation of chromatin function. Nucleic Acids Res. 2008;36(2):570-577.

9. Peng C, Lu Z, Xie Z, et al. The first identification of lysine malonylation substrates and its regulatory enzyme. Mol Cell Proteomics. 2011;10(12):M111.

10. Weinert BT, Schölz C, Wagner SA, et al. Lysine succinylation is a frequently occurring modification in prokaryotes and eukaryotes and extensively overlaps with acetylation. Cell Rep. 2013;4(4):842-851.

11. Lan F, Shi Y. Epigenetic regulation: methylation of histone and nonhistone proteins. Sci China C Life Sci. 2009;52(4):311-322.

12. Yang XD, Lamb A, Chen LF. Methylation, a new epigenetic mark for protein stability. Epigenetics. 2009;4(7):429-433.

13. Egorova KS, Olenkina OM, Olenina LV. Lysine methylation of nonhistone proteins is a way to regulate their stability and function. Biochemistry (Mosc). 2010;75(5):535-548. 
14. Stark GR, Wang Y, Lu T. Lysine methylation of promoterbound transcription factors and relevance to cancer. Cell Res. 2011;21(3):375-380.

15. Fischer EH, Graves DJ, Crittenden ER, et al. Structure of the site phosphorylated in the phosphorylase b to a reaction. J Biol Chem. 1959;234(7):1698-1704.

16. Phillips DM. The presence of acetyl groups of histones. Biochem J. 1963;87:258-263.

17. Lipmann F, Kaplan NO. Coenzyme for acetylation, a pantothenic acid derivative. J Biol Chem. 1947;167(3):869.

18. Allfrey VG, Faulkner R, Mirsky AE. Acetylation and Methylation of Histones and Their Possible Role in the Regulation of Rna Synthesis. Proc Natl Acad Sci USA. 1964;51:786-794.

19. Sun C, Wang M, Liu X, et al. PCAF improves glucose homeostasis by suppressing the gluconeogenic activity of PGC-1alpha. Cell Rep. 2014;9(6):2250-2262.

20. Lantier L, Williams AS, Williams IM, et al. SIRT3 is crucial for maintaining skeletal muscle insulin action and protects against severe insulin resistance in high fat fed mice. Diabetes. 2015;64(9):3081-3092.

21. Choi SW, Gatza E, Hou G, et al. Histone deacetylase inhibition regulates inflammation and enhances Tregs after allogeneic hematopoietic cell transplantation in humans. Blood. 2015;125(5):815-819.

22. Jaganathan A, Chaurasia P, Xiao GQ, et al. Coactivator MYST1 regulates nuclear factor-kappaB and androgen receptor functions during proliferation of prostate cancer cells. Mol Endocrinol. 2014;28(6):872-885

23. Schwer B, Bunkenborg J, Verdin RO, et al. Reversible lysine acetylation controls the activity of the mitochondrial enzyme acetyl-CoA synthetase 2. Proc Natl Acad Sci U S A. 2006;103(27):10224-10229.

24. Strahl BD, Allis CD. The language of covalent histone modifications. Nature. 2000;403(6765):41-45.

25. Sykes SM, Mellert HS, Holbert MA, et al. Acetylation of the p53 DNA-binding domain regulates apoptosis induction. Mol Cell. 2006;24(6):841-851.

26. Wang Q, Yakun Z, Chen Y, et al. Acetylation of metabolic enzymes coordinates carbon source utilization and metabolic flux. Science. 2010;327(5968):1004-1007.
27. Yang XJ, Seto E. Lysine acetylation: codified crosstalk with other posttranslational modifications. Mol Cell. 2008;31(4):449-461.

28. Patel J, Pathak RR, Mujtaba S. The biology of lysine acetylation integrates transcriptional programming and metabolism. Nutr Metab (Lond). 2011;8:12.

29. Prives C, Manley JL. Why is p53 acetylated? Cell. 2001;107(7):815-818.

30. Lu L, Li L, Lv X, et al. Modulations of hMOF autoacetylation by SIRT1 regulate hMOF recruitment and activities on the chromatin. Cell Res. 2011;21(8):1182-1195.

31. Verdin E, Ott M. 50years of protein acetylation: from gene regulation to epigenetics, metabolism and beyond. Nat Rev Mol Cell Biol. 2015;16(4):258-264

32. Allis CD, Berger SL, Cote J, et al. New nomenclature for chromatinmodifying enzymes. Cell. 2007;131(4):633-636.

33. Mellert HS, McMahon SB. hMOF, a KAT(8) with many lives. Mol Cell. 2009;36(2):174-175.

34. O'Brien T, Tjian R. Functional analysis of the human TAFII250 N-terminal kinase domain. Mol Cell. 1998;1(6):905-911.

35. Mujtaba S, He Y, Zeng L, et al. Structural mechanism of the bromodomain of the coactivator CBP in p53 transcriptional activation. Mol Cell. 2004; 13(2):251-263.

36. Richon VM, Emiliani S, Verdin E, et al. A class of hybrid polar inducers of transformed cell differentiation inhibits histone deacetylases. Proc Natl Acad Sci U S A. 1998;95(6):3003-3007.

37. Borah JC, Mujtaba S, Karakikes I, et al. A small molecule binding to the coactivator CREB-binding protein blocks apoptosis in cardiomyocytes. Chem Biol. 2011;18(4):531-541.

38. Mujtaba S, Zhou MM. Anti-viral opportunities during transcriptional activation of latent HIV in the host chromatin. Methods. 2010;53(1):97-101.

39. Delmore JE, Issa GC, Lemieux ME, et al. BET bromodomain inhibition as a therapeutic strategy to target c-Myc. Cell. 2011;146(6):904-917. 